\title{
QUAND L'ACTION PUBLIQUE DEVIENT NÉCESSAIRE : QU'A SIGNIFIÉ « RÉSOUDRE » LA CRISE DE L'AMIANTE?
}

\author{
EMMANUEL HENRY
}

$\mathrm{S}$ ans avoir toutes les caractéristiques d'une crise politique ${ }^{1}$, les épisodes de forte publicité connus par certains problèmes apparaissent placer le personnel dirigeant dans une situation où il a à répondre dans l'urgence à un problème constitué comme prioritaire. Dans une première approche, la gestion des problèmes occupant la « une » des médias d'information semble fortement influencée par cette forte publicité, puisque le personnel politique intervient souvent à son plus haut niveau pour annoncer des décisions radicales. L'objet de cet article est de montrer que les processus qui conduisent à une modification des rythmes et des formes de l'action publique ne sont cependant pas aussi linéaires que cette première impression pourrait le laisser croire et surtout que les différents secteurs des administrations concernées réagissent de façon différenciée à une forte publicité.

L'analyse de situations critiques met en évidence certaines caractéristiques générales de l'action publique qui apparaissent de façon beaucoup plus visible dans ce type de contextes. Elle permet ainsi d'aborder une série de problèmes qui touchent à la sociologie de l'action publique. Tout d'abord, loin de désigner une série cohérente d'actions et d'interventions, ce qui est perçu comme la réponse politique et administrative à un problème n'est bien souvent que l'amalgame d'actions hétérogènes qui entretiennent une relation spécifique et particulière avec tel ou tel aspect de ce qui, à un moment donné, s'impose comme un problème ${ }^{2}$. Ces actions s'inscrivent dans des séries ou des enchaînements qui répondent à des logiques distinctes liées à des exigences professionnelles spécifiques, aux modalités de fonctionnement et d'organisation de tel secteur administratif ou aux relations entretenues avec des acteurs extérieurs à l'administration (relations régulières avec les acteurs mobilisés, pression plus ou moins forte exercée par les journalistes, etc.). Elles mobilisent donc des savoirs et des instruments spécifiques et s'inscrivent dans des temporalités variées, sans pour autant renvoyer à un fonctionnement dépourvu de toute logique, comme celui décrit dans le modèle du « garbage can $»^{3}$.

La publicisation d'un problème, loin d'unifier ce processus de l'action publique et de le soumettre entièrement à des logiques exogènes, a, au contraire, des effets différenciés sur chacun de ces enchaînements. Comme nous le montrerons au cours de

1. Michel Dobry, Sociologie des crises politiques. La dynamique des mobilisations multi-

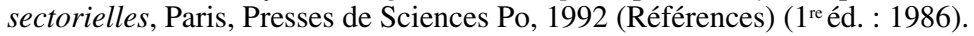

2. Cf. John W. Kingdon, Agendas, Alternatives and Public Policies, New York, Harper Collins, 1984.

3. Johan P. Olsen, James G. March, «Le modèle du "garbage can" dans les anarchies organisées », dans James G. March (dir.), Décisions et organisations, Paris, Les Éditions d'organisation, 1991 ( $1^{\text {re }}$ éd. anglaise : 1988), p. 163-204. 


\section{Emmanuel Henry}

cet article, elle conduit, de façon plus ou moins directe, à accélérer certains processus décisionnels ou à transformer profondément les pratiques routinisées de certains secteurs administratifs. Dans tous les cas, elle contraint le personnel politique à intervenir vigoureusement en transformant en décision « annonçable » ce qui est déjà largement instruit par l'administration, ce qui peut être facilement arbitré ou ce qui peut permettre une communication spectaculaire. Bien qu'elle aboutisse à la redéfinition et à la reconnaissance d'un problème, la publicisation ne fait donc pas pour autant disparaître la diversité des approches et des logiques existantes. Elle a cependant un certain nombre d'effets observables dans le rapprochement d'acteurs distincts, par des jeux d'anticipation, de mise en alerte, d'imposition de représentations de l'acceptable et de l'inacceptable, dans l'unification de temporalités propres à certains secteurs de l'action publique ou dans la modification de rapports de force entre acteurs intervenant à un titre ou à un autre dans les processus de décision.

L'influence de la publicité sur les processus politico-administratifs de gestion d'un problème sera analysée à partir de la crise induite par les conséquences sanitaires d'expositions à l'amiante. Sans exposer dans le détail l'ensemble des caractéristiques de ce problème, il est nécessaire de rappeler que ce minéral est le cancérogène professionnel le plus meurtrier, tuant plusieurs milliers de personnes par an, essentiellement parmi celles qui travaillent à son contact ${ }^{1}$. Cet aspect professionnel du problème est connu depuis la fin de la Seconde Guerre mondiale par un certain nombre d'acteurs touchés directement par une maladie, travaillant dans le domaine du risque professionnel ou intervenant dans le processus de décision publique à son propos. Il n'a, en revanche, pas suscité l'attention d'acteurs susceptibles de donner une diffusion sociale plus large à ce problème, comme les journalistes généralistes. Hormis un épisode de forte publicisation au milieu des années 1970, la question de l'amiante est quasiment absente des discours publics jusqu'en $1994^{2}$. Elle n'acquiert une dimension publique qu'à partir des années 1994-1995, quand le problème est publiquement redéfini à partir de ses prolongements environnementaux, en particulier autour de la présence d'amiante dans de nombreux bâtiments. Les aspects professionnels du risque amiante sont alors quasiment occultés des discours publics, bien qu'ils constituent l'essentiel du problème en termes de santé publique ${ }^{3}$. Ainsi, durant la période 1994-1996, l'amiante est publiquement présenté comme un toxique menaçant l'ensemble de la population, dont l'État n'a pas été capable ou n'a pas voulu interdire l'utilisation. Ces

1. Sur les aspects scientifiques du problème de l'amiante, cf. Institut national de la santé et de la recherche médicale (INSERM), Marcel Goldberg, Denis Hémon, rapporteurs, Effets sur la santé des principaux types d'exposition à l'amiante. Rapport établi à la demande de la Direction des relations du travail et de la Direction générale de la santé, Paris, INSERM, 1997 (Expertise collective).

2. Sur les raisons de cette faible publicité donnée à la question de l'amiante, cf. Emmanuel Henry, «Du silence au scandale. Des difficultés des médias d'information à se saisir de la question de l'amiante », Réseaux, 21(122), 2003, p. 237-272. Sur la mobilisation des années 1970 et ses répercussions médiatiques, cf. l'ouvrage publié par un des acteurs à la pointe de cette mobilisation, Collectif intersyndical sécurité des universités Jussieu CFDT/CGT/FEN, Danger! Amiante, Paris, François Maspéro, 1977 (Cahiers libres, 334).

3. Ce mécanisme général dans la presse nationale est particulièrement vrai pour les journaux télévisés puisque, de septembre 1995 à juin 1996, pendant la montée en puissance du problème, vingt-cinq reportages sur l'amiante sont diffusés par TF1, France 2, France 3 et M6, dans leurs journaux de début de soirée. Parmi ces reportages, seuls deux sont consacrés à la dimension professionnelle des pathologies. Tous les autres reportages concernent presque exclusivement le problème de la présence d'amiante dans des locaux recevant du public. 
discours dénonçant une nouvelle «affaire de santé publique » à la suite de celle du sang contaminé sont renforcés par le développement de procédures judiciaires et la mobilisation d'un certain nombre de structures associatives et, en particulier, d'associations de victimes. Une forte pression pèse alors sur le personnel politique, sommé d'intervenir et de prendre position.

Voici quelques points de repère chronologiques du développement de cette crise : à partir de mai 1995, ce problème s'impose comme médiatiquement central, avec une information régulièrement diffusée sur ce thème par l'ensemble des médias écrits et audiovisuels ; un processus de traitement administratif s'engage alors, qui aboutit à de premières décisions en février $1996^{1}$; en juin 1996, une plainte est déposée par l'Association nationale de défense des victimes de l'amiante (ANDEVA); le 3 juillet 1996, les premiers résultats de l'expertise collective menée par l'INSERM sont annoncés : elle évalue à plus de 2000 par an le nombre de décès imputables à l'amiante. Un certain nombre de décisions politiques sont alors annoncées (interdiction de l'amiante et annonce de la fermeture du campus de Jussieu), qui contribuent à une normalisation progressive du problème public.

Si les mobilisations suscitées par l'amiante et son intense couverture médiatique influencent le processus de sa gestion politico-administrative, celui-ci ne peut être simplement ramené à la solution trouvée au problème public qui s'est imposé en quelques mois. Analyser sa prise en charge par les acteurs politiques et administratifs comme le résultat direct d'une action hérö̈que de la part de quelques acteurs longtemps isolés, soutenus par une intense campagne médiatique, ne rend qu'imparfaitement compte de la réalité. De nombreuses autres logiques sont à prendre en considération, dont certaines restent assez indépendantes du processus de publicisation et de médiatisation. L'irruption du thème de l'amiante sur la scène publique modifie certainement les modalités de sa gestion, elle n'est pourtant qu'un élément parmi d'autres et seule l'analyse de la pluralité des facteurs qui entrent en ligne de compte peut permettre d'évaluer le poids réel de la publicité sur la prise en charge d'un problème.

Nous analyserons ainsi, dans un premier temps, les influences différentes de la publicité sur deux secteurs administratifs : nous montrerons tout d'abord comment la Direction générale de la santé (DGS) est amenée à prendre en compte le risque de constitution d'une crise dans sa gestion du problème, puis comment la Direction des relations du travail (DRT) n'est influencée que de façon médiatisée par ces processus. Nous étudierons enfin le positionnement spécifique du personnel politique face à un problème fortement publicisé et, en particulier, l'adéquation de son intervention à la problématisation publique, qui permet le retour à une certaine normalisation ${ }^{2}$.

1. En particulier, deux décrets pris simultanément le 7 février 1996, le premier par le ministère de la Santé, qui rend obligatoire le repérage de certains matériaux contenant de l'amiante dans les bâtiments, et le second par le ministère du Travail, qui renforce la réglementation sur l'usage professionnel de l'amiante.

2. Cet article est une version remaniée d'un chapitre de ma thèse (Emmanuel Henry, « Un scandale improbable. Amiante: d'une maladie professionnelle à une "crise de santé publique" », thèse de doctorat, Université de technologie de Compiègne, 2000). Il repose sur le même travail empirique, soit l'analyse des discours produits par la presse écrite et audiovisuelle et des décisions prises, et celle des entretiens effectués auprès des acteurs étant intervenus sur ce problème. Je tiens à remercier Vincent Dubois, Claude Gilbert et Pierre Muller pour leurs lectures de versions antérieures de ce texte et particulièrement Jacques Lagroye, non seulement pour ses relectures, mais pour avoir permis que ce travail voie le jour. 


\section{DE LA PRÉVENTION À LA PRÉCAUTION : LA MODIFICATION DES PRATIQUES ADMINISTRATIVES DE GESTION DES RISQUES SANITAIRES}

Avant 1994, lorsque l'amiante n'est pas encore l'objet d'une attention publique prioritaire, ce problème est abordé par les administrations centrales de façon routinière, principalement à travers le processus de rédaction d'un décret organisant le recensement des bâtiments contenant de l'amiante et le travail régulier d'incorporation en droit français de directives européennes. Du point de vue de l'administration, il se dédouble en deux problèmes principaux : le risque professionnel pris en charge au sein du ministère du Travail par la DRT et le risque pour la population générale, géré par la DGS, dépendant, selon les gouvernements, du ministère de la Santé ou de celui du Travail (lorsque la Santé est occupée par un secrétariat d'État dépendant de ce ministère) ${ }^{1}$. Même si, dès la constitution de la crise de l'amiante et la redéfinition du problème, tous les regards des journalistes se tournent vers la DGS et le ministère de la Santé, il faut tout de même souligner que l'essentiel des problèmes posés par l'amiante relève du ministère du Travail.

Les risques associés aux expositions à l'amiante confrontent les administrations centrales à deux problèmes de nature assez différente. L'un, le risque professionnel, est strictement une question de prévention. Il s'agit de gérer l'exposition des personnels concernés à un risque connu et scientifiquement bien documenté. Le second, le risque environnemental, principalement lié à l'amiante en place dans les bâtiments en direction des occupants, pose le problème du positionnement de l'administration face à des risques impossibles à mesurer et faibles. Si ces deux problèmes ne sont pas totalement séparables - la présence d'amiante dans les bâtiments pose aussi directement problème aux professionnels du bâtiment amenés à y intervenir -, ils permettent tout de même de distinguer deux types d'évolution dans le traitement des questions de santé, l'une liée à la généralisation du principe de précaution, la seconde touchant à un changement dans les modalités de gestion du risque professionnel au sein de l'administration du travail. Ces deux évolutions, aux conséquences directes sur la gestion des problèmes posés par l'utilisation de l'amiante, restent liées puisqu'elles sont, en partie, les conséquences directes de la succession de confrontations de l'administration à des problèmes nouveaux, la gestion des problèmes de santé publique dans un contexte de forte publicité, ce qu'il est convenu d'appeler les «crises de santé publique ». Celles-ci posent d'une façon nouvelle la question des liens entre sciences, expertise et décision publique ${ }^{2}$. L'émergence de l'amiante sur l'agenda politico-administratif doit donc s'analyser dans le cadre d'une évolution à long terme de l'approche des questions de santé publique par plusieurs secteurs administratifs.

1. D'autres secteurs administratifs sont évidemment aussi plus ou moins directement concernés par les problèmes liés à l'amiante, comme la Direction de la sécurité sociale sur les questions d'indemnisation, l'environnement, sur le problème de la gestion des déchets contenant de l'amiante. Celle du logement travaille aussi directement en lien avec la DGS sur l'élaboration des décrets concernant la présence d'amiante dans les immeubles.

2. Cf. François Ewald, «Le retour du malin génie. Esquisse d'une philosophie de la précaution », dans Olivier Godard (dir.), Le principe de précaution dans la conduite des affaires humaines, Paris, MSH-INRA, 1997, p. 99-126. 
L'attention nouvelle portée par l'administration et le personnel politique à la question de l'amiante ne peut pas s'analyser exclusivement comme un effet direct de la forte publicisation de ce problème spécifique. La référence à l'affaire du sang contaminé, présente dans la majeure partie des entretiens effectués auprès des gestionnaires confrontés à cette nouvelle crise, le montre : cette évolution est analysée comme un effet direct de la succession de crises de santé publique ayant précédé ou se déroulant parallèlement à celle de l'amiante ${ }^{1}$. Cet effet se traduit par l'intériorisation de la crainte d'avoir à rendre compte de ses décisions ou de ses non-décisions, soit, directement et en temps réel, face aux médias d'information, soit, indirectement, dans le cadre d'une procédure judiciaire qui pourrait être intentée par les victimes des conséquences de décisions prises - ou non - par l'administration.

Les crises de santé publique peuvent ainsi être analysées comme les signes de deux bouleversements majeurs. Le premier est la plus grande publicité accordée à ces problèmes. Elle s'observe bien sûr dans la part croissante, parfois prédominante, accordée par les médias d'information aux questions de santé ${ }^{2}$ et dans les nouvelles formes à travers lesquelles elles sont présentées par les journalistes ${ }^{3}$. Le second est la traduction judiciaire de plus en plus régulière de ces problèmes. Celle-ci est liée à une plus grande facilité de mobilisation des victimes, particulièrement évidente dans l'épidémie de sida, mais observable également dans le cadre d'autres types de maladies ou d'accidents ${ }^{4}$. Les associations de victimes hésitent de moins en moins à faire appel à la justice pour obtenir réparation des erreurs ou des dysfonctionnements qu'elles estiment être à l'origine de leurs souffrances. L'ensemble des personnels administratifs et politiques intériorise donc le risque d'être mis en cause par l'institution judiciaire pour la façon dont ils ont traité ou traitent une question de santé publique. Les questions de santé deviennent ainsi prioritaires et sont l'objet d'une attention et d'une vigilance extrêmes, tant de la part du personnel politique qu'administratif.

«Le gouvernement était... enfin, les ministres, son directeur de cabinet étaient trop échaudés par les affaires antérieures du sang contaminé pour envisager que ça recommence. Donc, c'était un dossier qu'on devait surveiller comme le lait

1. «L'affaire de la transfusion sanguine a précipité les choses au début des années 1990 ; puis l'année 1996 a marqué un pic paroxystique avec les crises de la vache folle, de l'hormone de croissance, de la maladie de Creutzfeld-Jakob, ou encore de l'amiante. L'année durant, la thématique de la sécurité sanitaire a occupé la une de la presse écrite et des journaux télévisés » (Jean-François Girard, Jean-Michel Eymeri, Quand la santé devient publique, Paris, Hachette Littératures, 1998, p. 105).

2. Cf. Michel Mathien (dir.), Médias, santé, politique, Paris, L'Harmattan, 1999 (Communication).

3. Dominique Marchetti, «Contribution à une sociologie des transformations du champ journalistique dans les années 1980 et 1990. À propos d' "événements sida" et du "scandale du sang contaminé" », thèse de doctorat, EHESS, 1997.

4. Cf. pour le cas du sida, Janine Barbot, Les malades en mouvement. La médecine et la science à l'épreuve du sida, Paris, Balland, 2002 (Voix et regards). Plus généralement, cf. Jacques Bresson, «Que veulent les associations de victimes? », dans Geneviève Decrop, JeanPierre Galland (dir.), Prévenir les risques : de quoi les experts sont-ils responsables ?, Paris, Éditions de l'Aube, 1998, p. 163-173 ou Jean-Paul Vilain, Cyril Lemieux, «La mobilisation des victimes d'accidents collectifs. Vers la notion de "groupe circonstanciel" », Politix, 44, 4 trim. 1998, p. 135-160. 


\section{Emmanuel Henry}

sur le feu et surtout sur lequel on n'avait pas le droit à l'erreur en quelque sorte. ${ }^{1}$

«Il y a le sang contaminé qui maintenant exige... Enfin, le fait que des gens de l'administration aient été mis en examen fait que maintenant, dans l'administration, tout le monde veille à ne pas pouvoir être mis en examen dans le cas de l'amiante... Donc, ça donne plus de rigueur, ce n'est pas plus mal... Mais beaucoup de stress aussi, c'est évident. » ${ }^{2}$

Les effets de la forte médiatisation d'un problème ne peuvent donc pas être mesurés en eux-mêmes, ils doivent être analysés en lien avec les autres effets attendus d'une forte mobilisation autour d'un problème et, en particulier, ses éventuelles conséquences judiciaires. Il est difficile d'évaluer par quels vecteurs passe cette pression ressentie par les acteurs, mais on peut émettre l'hypothèse que le fort investissement d'un problème par les médias d'information est aussi perçu comme une mobilisation forte des acteurs concernés, mobilisation dont les chances d'avoir des développements judiciaires sont de plus en plus importantes. La surface médiatique occupée par un problème, les mobilisations de victimes ou d'acteurs concernés, le risque d'une procédure judiciaire sont donc autant de facteurs qui contraignent les acteurs politiques et administratifs à redoubler de vigilance sur ces questions. Ces différentes dimensions des «crises de santé publique » sont indissociables dans leurs effets puisqu'elles se cumulent et se renforcent les unes les autres.

Cette nouvelle attitude doit aussi s'analyser en lien avec une évolution plus générale qui marque une rupture dans les attitudes sociales et politiques face aux risques, et qui peut être résumée par le recours de plus en plus régulier au «principe de précaution ». Pourtant, loin de séparer strictement deux périodes, le passage de la prévention à la précaution révèle plutôt un chevauchement entre deux approches différentes du risque, de la science, de l'expertise et de la décision publique. Le domaine du risque professionnel est, par exemple, plus tardivement touché par cette évolution, qui concerne, en premier lieu, les domaines de l'environnement et de la santé publique pour les populations générales. De plus, si certaines définitions jurisprudentielles du principe de précaution sont assez explicites ${ }^{3}$, elles n'aboutissent pas pour autant à constituer un principe qui serait sans équivoque dans sa formulation et sans ambivalence dans son application ${ }^{4}$. Ce principe peut tout d'abord être spontanément appréhendé sous la forme d'un simple «précepte de bon sens » du type : « dans le doute, abstiens-toi » ${ }^{5}$, devenant ainsi disponible pour des usages dans des acceptions diffé-

1. Entretien avec un membre du cabinet de Jacques Barrot au ministère du Travail et des Affaires sociales, inspecteur général des Affaires sociales, 8 février 1999.

2. Entretien avec le chef du Bureau hygiène en milieu de travail, Direction des relations du travail, 13 janvier 1999.

3. «Il appartenait à l'autorité administrative [...] d'interdire, sans attendre d'avoir la certitude que tous les lots de produits dérivés du sang étaient contaminés, la délivrance des produits dangereux », arrêt du Conseil d'État du 9 avril 1993, cité dans Marie-Angèle Hermitte, Le sang et le droit. Essai sur la transfusion sanguine, Paris, Le Seuil, 1996, p. 309, souligné par l'auteur. La formulation du commissaire du gouvernement, dans ses conclusions pour cette décision, est encore plus explicite quand il écrit : «En situation de risque, une hypothèse non infirmée devrait être tenue, provisoirement, pour valide, même si elle n'est pas formellement démontrée », ibid., p. 310.

4. Olivier Godard, "L'ambivalence de la précaution et la transformation des rapports entre science et décision », dans Olivier Godard (dir.), op. cit., p. 46.

5. Claude Gilbert, avec Isabelle Bourdeaux, «La précaution dans "l'empire du milieu" », dans Olivier Godard (dir.), op. cit., p. 312. 
rentes au sein d'espaces sociaux très divers. Mais, même en se restreignant à ses usages au sein des administrations publiques, il est difficile de trouver une modalité de son application qui serait une traduction pure et simple de ce principe. La raison principale tient à la nature de l'action publique, qui, en tant qu' action collective organisée, ne peut pas être analysée simplement comme la mise en œuvre univoque de principes juridiques ${ }^{1}$. L'introduction du principe de précaution doit donc plus justement s'analyser comme la mise en place de nouvelles procédures et de nouvelles modalités de négociation entre les différentes catégories d'acteurs parties prenantes dans les processus qui mènent aux décisions publiques ${ }^{2}$.

Le cas de l'amiante permet d'observer plusieurs types d'appropriation du principe de précaution par différents secteurs administratifs. Si ces variations tiennent à la nature différente des risques qu'ils ont à gérer, elles sont aussi induites par des fonctionnements spécifiques à la DGS et à la DRT, qui ont des formes d'organisation et d'appréhension des problèmes très différenciées.

\section{GÉNÉRALISATION DU PRINCIPE DE PRÉCAUTION À LA DGS}

Le problème que doit traiter la Direction générale de la santé répond à une application du principe de précaution proche de sa définition la plus évidente. Face à un doute quant à la toxicité à faible dose des expositions passives à l'amiante en place dans les bâtiments, quel type de décision mettre en œuvre ? Convient-il de privilégier l'existence d'un doute face à un risque si faible qu'il ne pourra jamais être définitivement mis en évidence scientifiquement ? Ou faut-il privilégier une solution de protection maximale des populations ? C'est autour de ces questions que se structure progressivement l'intervention de la DGS. Pour ses responsables, le problème réapparaît avec les mobilisations autour des craintes provoquées par la présence de flocages à base d'amiante à la fin des années 1980. Jusqu'à cette date, les risques liés à l'amiante, essentiellement professionnels, sont perçus comme ne relevant pas de leur compétence.

«Jusqu'en [19]94, c'est vrai que le problème de l'amiante était un problème vécu, connu et, je crois, assez unanimement considéré comme un problème de risque professionnel, que les risques professionnels, ça, c'est le code du travail avec une longue, une longue tradition. [...] Je crois que c'est ça qui était dominant : un, l'amiante, c'était d'ordre professionnel en termes sanitaires, et, deux, tout ce qui est risque professionnel est du domaine de la Direction du travail et du code du travail. $»^{3}$

Le risque pour la population générale réapparaît sur l'agenda administratif à travers la question du recensement des locaux contenant de l'amiante. Elle fait l'objet d'un traitement à long terme, le dossier étant expertisé par un organisme consultatif placé auprès de la DGS, le Conseil supérieur d'hygiène public de France (CSHPF). La

1. Cf. Pierre Lascoumes, «Normes juridiques et mise en œuvre des politiques publiques », L'Année sociologique, 40 (1), 1990, p. 43-71, et « Les arbitrages publics des intérêts légitimes en matière d'environnement. L'exemple des lois Montagne et Littoral », Revue française de science politique, 45 (3), juin 1995, p. 396-419.

2. Cf. Pierre Lascoumes, «La précaution comme anticipation des risques résiduels et hybridation de la responsabilité », L'Année sociologique, 46 (2), 1996, p. 359-382.

3. Entretien avec le directeur général de la santé de 1986 à 1997, 24 novembre 1998. 
reprise du dossier en 1994 par la DGS ne signifie donc pas que l'amiante n'était pas traité dans ce service avant cette date, mais simplement qu'il y était appréhendé comme un problème mineur à travers des routines administratives traditionnelles. Dans ce contexte, le décret annoncé par la ministre de la Santé en mai 1995 et finalement publié le 7 février $1996{ }^{1}$ est le résultat d'un processus d'élaboration réglementaire dont la logique est essentiellement issue d'un fonctionnement autonome de l'administration. Il marque toutefois un certain infléchissement, dans le sens où il engage vers des mesures contraignantes face à un risque faible.

S'il peut être analysé comme une application du principe de précaution, il faut toutefois souligner que les décisions prises apparaissent assez corrélées avec la dimension publique prise par le problème. En effet, face à un risque perçu comme extrêmement faible, c'est dans ses éventuels prolongements publics qu'il faut chercher les raisons qui poussent les acteurs administratifs à intervenir. Sans conclure à un lien direct entre médiatisation et prise en charge publique, le risque d'une forte publicité du dossier, parce qu'il révèle une forte mobilisation des publics concernés, suscite une attention supplémentaire. Plusieurs indices concordent pour valider cette hypothèse. Tout d'abord, le premier acteur proche des instances de décisions publiques à se soucier du problème est le Comité permanent amiante (CPA), une instance organisée par les industriels du secteur et rassemblant les principaux intervenants sur cette question, dont une des préoccupations majeures est la peur que se reproduisent de fortes mobilisations proches de celles de 1975 ou une crise dans l'opinion ${ }^{2}$.

Ensuite, il faut noter que l'attention du personnel politique se concentre particulièrement sur les risques encourus par la population générale et, plus précisément, par les populations perçues comme les plus vulnérables - dans le cas de l'amiante, les enfants dans les écoles ${ }^{3}$. Non seulement ces aspects du problème doivent être traités avec une attention spécifique, mais les décisions prises à ce sujet doivent faire l'objet d'un affichage politique fort à l'attention de l'opinion. Ainsi, dans le décret de février 1996, la priorité est donnée aux établissements d'enseignement, crèches et éta-

1. Décret 96-97 du 7 février 1996, Journal officiel, 8 février 1996, p. 2049-2050. Ce décret rend obligatoire, pour tous les propriétaires privés et publics d'immeubles collectifs construits avant le $1^{\text {er }}$ janvier 1980, la recherche des flocages et des calorifugeages contenant de l'amiante.

2. L'importance accordée aux effets sur l'opinion publique du problème des flocages apparaît dans de nombreux comptes rendus de réunions, comme lors de celle du 16 mai 1989: «Constatant que le risque pour la santé publique n'était pas aussi grave qu'on aurait pu le croire, le milieu scientifique a pris un certain recul sur ce dossier. Bien sûr, des risques demeurent, mais ils ne semblent pas inquiétants. Il n'est toutefois pas question de les ignorer et les conseils de prévention du CPA sont à ce titre très importants pour gérer le présent et l'avenir. Le plus préoccupant, en fait, serait une flambée soudaine de l'opinion publique qui se solderait par des décisions hâtives et des actions incontrôlées, potentiellement dangereuses. C'est pourquoi, le CPA doit, absolument, persévérer dans sa politique de contrôle et de prévention », compte-rendu de la réunion plénière du CPA du 16 mai 1989, disponible sur le site du ministère de la Santé < http://www.sante.gouv.fr/amiante/connaitre/histoire/document/textes.htm > [souligné par moi].

3. Un phénomène similaire est analysé par Jean Gustave Padioleau au sujet de la consommation de tabac : "L'intervention apparaît d'autant plus légitime qu'elle vise en premier des citoyens auprès de qui l'État se pose volontiers en ange tutélaire, les femmes, en particulier, les femmes enceintes, les adolescents et les enfants. La perception des coûts sociaux s'accompagne enfin d'efforts de promotion des coûts individuels négatifs subis par les "fumeurs passifs" - les non-fumeurs se trouvant au voisinage d'un fumeur ou dans une atmosphère enfumée » (Jean Gustave Padioleau, « La lutte contre le tabagisme : action politique et régulation étatique de la vie quotidienne », L'État au concret, Paris, PUF, 1982 (Sociologies), p. 54$55)$. 
blissements hébergeant des mineurs. Dans ces bâtiments, le recensement de l'amiante doit être effectué dans des délais beaucoup plus courts.

Une décision est particulièrement révélatrice de la difficulté à dissocier décision de santé publique et décision de santé à destination de l'opinion publique. Il s'agit du décret du 26 juillet $1996^{1}$. Alors que les restrictions d'utilisation de l'amiante étaient, jusqu'à ce décret, exclusivement la transposition de directives européennes, la première décision qui trouve son origine dans les services politico-administratifs français concerne les produits de consommation courante, dont la mise en cause a été la plus forte dans les discours publics sur l'amiante. Sur la liste des produits contenant de l'amiante, interdits par ce nouveau décret, vient en première position le grille-pain, suivi de près par les housses de tables à repasser et les repose-fer - objets qui ont abondamment servi d'illustration au problème de l'amiante dans les discours médiatiques. L'importance accordée à ce texte dans le rapport de l'Office parlementaire d'évaluation des choix scientifiques et technologiques et le regret quant à sa confidentialité montre que son objectif est double. S'il répond à la volonté d'éliminer tout risque, il est aussi un signal de l'importance accordée aux problèmes qui ont le plus attiré l'attention du public au cours de cette crise, du fait de leur importance dans les discours médiatiques d'information ${ }^{2}$.

On trouve enfin un troisième indice de la corrélation entre la dimension publique acquise par une question et l'accélération de sa prise en charge effective en comparant l'amiante à des toxiques posant des problèmes similaires pour ce service administratif. On observe alors de nettes distorsions dans la façon dont sont gérés les dossiers, selon la surface publique et médiatique qu'ils occupent ou sont susceptibles d'occuper.

«C'est clair que les ministres se méfient beaucoup plus du problème de l'amiante que du problème du plomb parce qu'ils ont très peur des retombées médiatiques de toutes les décisions qui peuvent être prises ou de toutes les décisions qui peuvent ne pas avoir été prises. [...] Soit c'est un dossier très sensible et, à ce moment-là, c'est géré directement par les ministres, soit ce ne sont pas des dossiers sensibles et on ne fait rien sortir parce que, de toute façon, ça n'intéresse personne. $»^{3}$

Les effets de la publicité passent ainsi principalement par des demandes de plus en plus régulières de la part des cabinets ministériels auprès des services administratifs. Ils aboutissent à privilégier des décisions qui permettent un affichage fort et res-

1. Décret 96-668, Journal Officiel, 27 juillet 1996, p. 11432-11433.

2. «Attendu depuis longtemps et dans la ligne de la décision du 3 juillet [jour de l'annonce de l'interdiction de l'amiante en France], le décret n 96-668 du 26 juillet 1996 relatif aux produits contenant de l'amiante complète la liste des interdictions existantes. [...] Désormais sont interdits les produits à usage domestique suivants : grille-pain, dispositifs de répartition de chaleur, tables à repasser, housses de tables à repasser, repose-fer, appareils de chauffage mobiles, panneaux isolants destinés au bricolage. [...] On ne peut que se féliciter de la parution de ce texte qui permet de supprimer l'amiante de notre environnement immédiat. [...] On peut regretter la parution d'un tel texte au J.O. du 27 juillet 1996 et donc l'extrême confidentialité qui a entouré sa sortie » (Office parlementaire d'évaluation des choix scientifiques et technologiques (OPECST), «L'amiante dans l'environnement de l'homme: ses conséquences et son avenir », rapport édité par l'Assemblée nationale (329) et le Sénat (41), Paris, 1997, p. 49-50).

3. Entretien avec le chef du Bureau veille sanitaire, risques du milieu et alimentation, Direction générale de la santé, 18 mars 1999. Sur la question du plomb, cf. Christine Dourlens, Saturnisme infantile et action publique, Paris, L'Harmattan, 2003 (Risques collectifs et situations de crise). 
taurent une image positive de l'action politique. La prise en charge du risque amiante à destination de la population générale reste donc assez corrélée à la dimension publique réelle et potentielle de ce problème. La surface publique et médiatique que ce risque est susceptible d'occuper compte donc parmi les facteurs qui expliquent le processus de son traitement administratif.

\section{«RISQUE ACCEPTABLE » ET PRÉCAUTION : LA DRT CONFRONTÉE AU RISQUE AMIANTE}

Le changement de perspective est assez différent au sein de la DRT et du ministère du Travail, puisque le problème qui se pose ici est tout autre. Plus qu'à la prise en compte nouvelle d'un risque auparavant négligé, c'est progressivement une nouvelle approche du risque professionnel qui est développée vis-à-vis de l'amiante. La précaution se décline alors dans des formes assez différentes, puisqu'elle vise non à éradiquer, mais à limiter « autant que possible» un risque connu ${ }^{1}$. C'est donc la question de l' «acceptabilité » du risque qui est posée. Le risque « acceptable » ne peut toutefois pas être défini autrement que comme un risque socialement accepté, c'est-à-dire un risque justifié aux yeux des acteurs intervenant dans le processus décisionnel et imposé avec succès aux acteurs qui en subissent les conséquences. Il est donc, en premier lieu, le résultat de rapports de forces entre acteurs et groupes d'acteurs ayant à le définir et/ou l'accepter et le subir. Or, ces rapports de forces sont considérablement modifiés avec la reprise des mobilisations sur la question de l'amiante.

Les conséquences de la publicisation apparaissent toutefois beaucoup moins directement sur la DRT que sur la DGS. Deux caractéristiques rendent en effet la DRT moins perméable que la DGS aux changements de définition publique du problème. La première tient à la forte intégration européenne de la réglementation du risque professionnel qui contraint les agents du ministère sur le plan du rythme et du contenu des différentes réformes réglementaires. Ainsi, lorsque l'amiante réapparaît sur l'agenda administratif, le problème de l'usage d'un cancérogène professionnel ne peut plus être traité de la même manière qu'auparavant depuis l'entrée en vigueur d'une nouvelle directive européenne ${ }^{2}$. La deuxième caractéristique tient au fait que l'élaboration de la réglementation sur la santé au travail fait l'objet d'un processus de concertation sociale dans lequel interviennent les représentants d'organisations syndicales de salariés et d'employeurs, en particulier avec la consultation sur tous les textes du Conseil

1. C'est dans l'industrie nucléaire que cette déclinaison du principe de précaution est la plus ancienne et la plus formalisée, avec les recommandations de la Commission internationale de protection radiologique (CIPR). Celles-ci reposent sur trois principes : la justification de l'activité, l'optimisation de la protection (principe du ALARA, "As Low As Reasonably Achievable») et la limitation des doses individuelles. Cf. Philippe Hubert, « À la recherche du risque acceptable: enjeux autour d'une relation dose-effet», Culture technique, 11, septembre 1983, p. 93-101.

2. Il s'agit de la directive 90/394/CEE du 28 juin 1990 « concernant la protection des travailleurs contre les risques liés à l'exposition à des agents cancérigènes au travail », Journal Officiel des Communautés européennes, 26 juillet 1990, L196, p. 1-7. Ce texte énonce le principe de substitution d'un agent cancérogène « dans la mesure où cela est techniquement possible, par une substance, une préparation ou un procédé qui, dans ses conditions d'emploi, n'est pas ou est moins dangereux pour la santé ou, le cas échéant, pour la sécurité des travailleurs ». 


\section{Résoudre la crise de l'amiante?}

supérieur de prévention des risques professionnels (CSPRP), organisme consultatif dans lequel siègent toutes les organisations représentatives ${ }^{1}$.

\section{LA REPRISE DE L’INITIATIVE PAR LES RESPONSABLES DE LA DRT}

La première évolution visible à la DRT est une modification du positionnement de ses responsables administratifs vis-à-vis de leurs principaux interlocuteurs. Alors qu'en temps normal, ils se situent dans un certain retrait ${ }^{2}$, laissant l'initiative aux partenaires sociaux consultés ou aux impératifs européens, ils vont jouer un rôle moteur sur la question de l'amiante. La première manifestation de ce nouveau positionnement passe par la constitution d'une expertise autonome qui permet à ces acteurs d'acquérir un nouveau poids dans les rapports de force établis avec leurs partenaires.

La place laissée à la connaissance scientifique des dangers d'un toxique dans les modalités d'arbitrage propres à la DRT est longtemps restée assez faible : jusqu'en 1994, les processus décisionnels mêlent inextricablement connaissance scientifique du danger et arbitrages socio-économiques, tout en se déroulant dans une relative opacité. L' « usage contrôlé de l'amiante » correspond à un choix opéré en fonction de certains déterminants économiques, sans avoir nécessité le développement d'une expertise ou d'une connaissance scientifique approfondie de ses conséquences ${ }^{3}$. L'absence de connaissances scientifiques stabilisées ne pose alors pas de problème puisque l'expertise sert essentiellement à légitimer et justifier le choix politique, d'ores et déjà effectué, d'usage d'un toxique ${ }^{4}$. Ce choix a d'ailleurs peu de chance d'être remis en cause sans une modification durable du rapport de force entre acteurs. Incluses dans le processus de négociation entre les différents partenaires, les connaissances scientifiques servent essentiellement d'armes dans les différentes discussions, sans permettre d'aboutir à un accord minimal sur les bases à partir desquelles il convient de prendre les décisions ${ }^{5}$. Le principal effet de la diffusion du principe de précaution face à un risque connu est

1. Sur les logiques de fonctionnement de la commission des maladies professionnelles du CSPRP, cf. Marc-Olivier Déplaude, «Codifier les maladies professionnelles : les usages conflictuels de l'expertise médicale », Revue française de science politique, 53 (5), octobre 2003, p. 707-735.

2. Retrait fortement contraint par les faibles moyens (en personnel et en capacité d'expertise) dont disposent les agents de ce bureau de la DRT.

3. «Dans la pratique française d'élaboration des normes et prescriptions de protection, il nous semble que s'effectue un "brouillage" entre les aspects proprement scientifiques de détermination du seuil d'innocuité et les considérations d'ordre économique (ou/et relevant des valeurs sociales), notamment au travers des multiples comités consultatifs répandus auprès de l'administration » (Jean-Paul Moatti, Économie de la sécurité. De l'évaluation à la prévention des risques technologiques, Paris, INSERM-La Documentation française, 1989 (Analyses et prospective), p. 24).

4. Sur ce point, cf. ce que Philippe Roqueplo appelle l' « expertise confisquée » à partir de l'exemple du nucléaire (Philippe Roqueplo, «L'expertise scientifique : convergence ou conflit de rationalités ? », dans Jacques Theys, Véronique Liber, Marie-Pierre Palacios (dir.), Environnement, science et politique. Les experts sont formels, Paris, Germes, 1991 (Cahier. 13), p. 43 80). Cf. aussi Philippe Roqueplo, Entre savoir et décision, l'expertise scientifique, Paris, INRA, 1997 (Sciences en question).

5. Sur ces points, cf. les contributions rassemblées dans CRESAL, Les raisons de l'action publique. Entre expertise et débat. Actes du colloque CRESAL-CNRS, Saint-Étienne, 13-14 mai 1992, Paris, L'Harmattan, 1993 (Logiques politiques), en particulier «Discuter l'action publique en public. Confiscation technicienne ou professionnalisation du débat », p. 245-338. 


\section{Emmanuel Henry}

de remettre en cause les procédures par lesquelles ces choix sont effectués, en imposant plus de transparence et de clarté dans ces arbitrages ${ }^{1}$. Ceci est particulièrement vrai dans le cas des cancérogènes pour lesquels il est scientifiquement impossible de définir un seuil en deçà duquel tout risque pourrait être éliminé ${ }^{2}$. Un nouveau statut doit être donné à la connaissance scientifique du problème dans le processus de prise de décision.

«On a affiché maintenant tout à fait officiellement qu'on ne recherchait plus automatiquement le consensus, c'est-à-dire qu'on voulait avoir un point des données scientifiques, une concertation pour éclairer l'administration, mais que c'était à l'administration de trancher dans le sens qui lui paraissait le plus près de sa ligne politique et en fonction des données scientifiques et des éléments de dialogue social. $»^{3}$

Les difficultés à organiser une expertise indépendante des différents acteurs en présence montrent que la mise en place d'un processus de décision fondé sur une connaissance scientifique unanimement acceptée est une véritable nouveauté à la DRT et ne va pas sans poser de problème. La première étape de ce processus est l'organisation, en décembre 1994, d'une réunion rassemblant l'ensemble des « experts » travaillant, plus ou moins directement, sur le problème de l'amiante. Si elle a lieu en même temps que les premières apparitions publiques du problème, elle apparaît plutôt motivée par un ensemble de facteurs ou d'indices convergents qui entrent en contradiction avec l'approche antérieure du problème, centrée sur les seuls salariés d'usines utilisatrices ou transformatrices d'amiante. La publication à venir d'une étude épidémiologique anglaise qui souligne les risques encourus par les professionnels intervenant simplement sur des matériaux contenant de l'amiante - au premier rang desquels les ouvriers du bâtiment - provoque un débat dans certains secteurs de la santé au travail ${ }^{4}$. Plusieurs associations se mobilisent alors pour demander aux fonctionnaires du ministère du Travail, avec lesquels ils sont en relation régulière, une refonte de la réglementation, dans le but de protéger cette population.

La recherche d'un accord sur une connaissance des méfaits de l'amiante fait entrer la contradiction dans un domaine où, jusqu'alors, le processus d'établissement des rapports de forces s'opérait sans débat sur l'expertise, c'est-à-dire à travers le choix implicite des experts écoutés par le ministère du Travail et intervenant au nom de l'une ou l'autre des délégations entendues au cours des consultations préalables à toute décision. Le processus de constitution d'une expertise autonome aboutit à la art. cité.

1. Cf. Pierre Lascoumes, «La précaution comme anticipation des risques résiduels... »,

2. Cf. Catherine Le Galès, André Oudiz, «Contribution méthodologique à la détermination de valeurs limites d'exposition professionnelle à l'amiante. Relation exposition-risque et critères économiques », Revue d'épidémiologie et de santé publique, 32 (2), 1984, p. 113-121 ; Catherine Le Galès, André Oudiz, Prévention des cancers professionnels. Problèmes et prévention, Paris, Doin-INSERM, 1989 (Grandes enquêtes en santé publique et épidémiologie), et Jean-Paul Moatti, Yves Bonvalot, «Incertitudes scientifiques et gestion des risques pour la santé. L'exemple des toxiques cancérigènes », dans Jacques Theys, Véronique Liber, MariePierre Palacios (dir.), Environnement, science et politique. Les experts sont formels, Paris, Germes, 1991 (Cahier. 14), p. 141-166.

3. Entretien avec le chef du Bureau hygiène en milieu de travail, Direction des relations du travail, 13 janvier 1999.

4. Julian Peto, John T. Hodgson, Fiona E. Matthews, Jacqueline R. Jones, « Continuing Increase in Mesothelioma Mortality in Britain », The Lancet, 345 (8949), 4 mars 1995, p. 535539. 
demande officielle d'une expertise collective à l'INSERM, qui rompt définitivement avec l'approche antérieure du problème au sein de la DRT. Les principales conclusions du rapport d'expertise collective sont rendues publiques le 3 juillet 1996. Au-delà d'une estimation de la mortalité imputable à l'amiante à 2000 morts par an, le rapport souligne l'absence de seuil d'innocuité et le danger de toutes les sortes d'amiante ${ }^{1}$. Malgré les discussions auxquelles donne lieu la distinction entre établissement et gestion du risque ${ }^{2}$ et en acceptant que toute procédure d'évaluation d'un risque engage plus ou moins explicitement des valeurs sociales ${ }^{3}$, on peut tout de même affirmer, à la suite de Ulrich Beck, que «la science devient de plus en plus nécessaire, mais de moins en moins suffisante à l'élaboration d'une définition socialement établie de la vérité » ${ }^{4}$. Évoquer l'introduction d'une procédure d'expertise dans le processus décisionnel de l'administration n'implique pas que l'on évacue les interrogations sur le statut des opinions d'expert ou sur leur caractère socialement et historiquement situé ${ }^{5}$. Toutefois, ces questionnements supposent un préalable selon lequel les décisions politiques et administratives s'appuient sur une expertise scientifique. Or, c'est justement ce retournement que l'on observe au niveau de la DRT durant cette période.

Sans aboutir à constituer une connaissance scientifique totalement objective ou socialement neutre, la procédure d'expertise collective enclenche une dynamique d'accroissement et de diffusion de savoirs et de connaissances qui étaient longtemps restés confinés dans des arènes de discussion restreintes. La décision de faire appel à une expertise autonome permet donc aux acteurs du ministère de s'appuyer sur une définition scientifique du problème qui s'impose à l'ensemble des acteurs intervenant dans les négociations. À travers cette crise de l'amiante se mettent en place des procédures nouvelles qui s'appliquent dorénavant à un ensemble de toxiques professionnels ${ }^{6}$.

Le ministère du Travail se donne ainsi les moyens de sortir de la dépendance qui le liait aux expertises majoritairement produites en liaison avec les employeurs. Ce nouveau positionnement des acteurs politico-administratifs est grandement facilité par l'abandon du terrain par les industriels du secteur, qui savent la partie perdue ${ }^{7}$. Leur ligne de défense, centrée autour de la moindre nocivité du chrysotile, une des catégo-

1. INSERM, Effets sur la santé...,op. cit.

2. La distinction entre «risk assessment » et «risk management » a été, par exemple, âprement remise en cause par Mary Douglas et Aaron Wildavsky, «Assessment is Biased », dans Risk and Culture: An Essay on the Selection of Technical and Environmental Dangers, Berkeley, Los Angeles, Londres, University of California Press, 1982, p. 67-82.

3. "[The] main modes of risk assessment clearly reflect the social values of the assessors », ibid., p. 71.

4. Ulrich Beck, La société du risque. Sur la voie d'une autre modernité, Paris, Aubier, 2001 (Alto) (1 ${ }^{\text {re }}$ éd. allemande : 1986), p. 343-344, souligné par l'auteur.

5. Cf. Arie Rip, Peter Groenewegen, «Les faits scientifiques à l'épreuve de la politique », dans Michel Callon (dir.), La science et ses réseaux. Genèse et circulation des faits scientifiques, Paris, La Découverte, 1988, (Textes à l'appui), p. 149-172.

6. Par la suite, des expertises collectives seront à nouveau demandées sur d'autres toxiques professionnels, comme le plomb ou les éthers de glycol. Sur la mise en place de nouvelles procédures d'expertise dans l'administration, cf. Pierre-Benoît Joly, « Besoin d'expertise et quête d'une légitimité nouvelle : quelles procédures pour réguler l'expertise scientifique ? ", Revue française des affaires sociales, 1, janvier-mars 1999, p. 45-52.

7. Au moins en Europe occidentale, puisque les groupes industriels producteurs d'amiante déploient leurs activités dans des pays moins développés aux législations sociales moins contraignantes, cf. Annie Thébaud-Mony, L'envers des sociétés industrielles. Approche comparative franco-brésilienne, Paris, L'Harmattan, 1990. 
ries d'amiante, et d'une dénégation du risque aux faibles doses, s'effondre littéralement avec la publicité donnée aux travaux de l'INSERM.

«On a travaillé beaucoup avec eux [les industriels], mais dans un rapport qui était très clair. C'est-à-dire qu'on prenait des mesures dont ils ne voulaient pas et qu'on leur a toujours dit qu'elles n'étaient pas négociables. [...] À partir du moment où $[\ldots]$ ils ont été convaincus de la volonté des pouvoirs publics, ils ont absolument tout lâché. » ${ }^{1}$

De la part des représentants patronaux, ce durcissement de la position de l'administration tranche radicalement avec l'attitude plus consensuelle qui prévalait antérieurement, elle est surtout interprétée comme un effet direct de la forte publicité faite au problème.

«Maintenant, on n'a plus la même sérénité dans les commissions qu'on a pu avoir auparavant parce que non seulement il y a la difficulté des sujets, mais en plus, il y avait sur l'histoire de l'amiante une médiatisation qui... on ne va pas... heureusement que dans une démocratie, les journalistes... mais en même temps, ça rendait le débat encore plus difficile. $»^{2}$

\section{NOUVELLES RÈGLES DU JEU ET NOUVEAUX ÉQUILIBRES AU SEIN DE LA DRT}

En quelques mois, la Direction des relations du travail procède à la refonte complète de la réglementation en matière d'utilisation professionnelle de l'amiante. La principale innovation apportée par le décret du 7 février $1996^{3}$ est un élargissement des secteurs professionnels couverts par la réglementation protectrice, qui en définit dorénavant trois, dont celui des salariés intervenant sur des matériaux ou appareils susceptibles de libérer des fibres d'amiante. Plus qu'une simple modification de la réglementation antérieure, ce texte peut, au contraire, être considéré comme la première révision complète de la réglementation en vigueur depuis 1977.

$\mathrm{Si}$, comme on l'a vu, le nouveau positionnement des responsables de la DRT face à leurs partenaires joue un rôle central, comment peut-on analyser l'influence de la publicité sur la prise en charge du problème ? Pour les personnels politiques et administratifs, la santé au travail ne représente pas le même risque de provoquer une crise publique importante, ce domaine d'intervention n'étant suivi que d'assez loin par les médias d'information généralistes. Si leur action est corrélée à la forte publicisation de l'amiante, c'est plutôt par un risque de contagion des autres aspects du risque amiante sur le risque strictement professionnel. Au niveau de l'entrée du problème sur l'agenda des problèmes à traiter par l'administration, les effets de la publicité passent par deux vecteurs principaux : l'attention nouvelle portée à ces questions par le personnel politique et les effets de mobilisations d'acteurs militant pour une meilleure prise en compte du risque professionnel.

L'attention du personnel politique aux questions liées à l'amiante s'exprime, on l'a vu, par un plus grand intérêt porté, en général, aux questions de santé. Toutefois,

1. Entretien avec le sous-directeur chargé des conditions de travail, Direction des relations du travail, 24 mars 1999.

2. Entretien avec le responsable de la Commission santé et sécurité, MEDEF (ex-CNPF), 3 août 1999.

3. Décret 96-98 « relatif à la protection des travailleurs contre les risques liés à l'inhalation de poussières d'amiante », Journal Officiel, 8 février 1996, p. 2050-2053. 
des effets extrêmement directs d'une forte médiatisation peuvent aussi être observés. C'est le cas, par exemple, avec un reportage diffusé dans l'émission «Envoyé spécial » sur France 2, le 28 septembre 1995, qui montre le caractère particulièrement exposé de certains postes de travail dans les usines transformatrices ${ }^{1}$. La présence de la ministre de la Santé sur le plateau montre un circuit extrêmement court entre publicisation et prise en charge politique, comme le révèle le dialogue entre la ministre et le journaliste responsable de l'émission :

«Élisabeth Hubert: On a vu dans ce reportage des problèmes qui concernaient les professionnels, le milieu professionnel. Tout existe à l'heure actuelle dans ce domaine.

Bernard Benyamin : Mais tout n'est pas appliqué.

Élisabeth Hubert: Mais ce n'est pas appliqué et, ça, c'est inacceptable. Les images montrant des ouvriers manipulant l'amiante sans protection, c'est contraire à la réglementation. Et avec notre, mon collègue, Jacques Barrot, ministre du Travail, nous donnons, dans les jours à venir, des instructions à l'inspection du travail et à la médecine du travail, notamment dans les sites où l'on sait qu'il y a des entreprises de transformation et de fabrication de produits dans lesquels il y a de l'amiante, pour que la réglementation soit appliquée dans toute sa sévérité... ${ }^{2}$

Dans les jours qui suivent cette émission, pas moins de sept visites de l'inspection du travail ont lieu dans l'usine incriminée ${ }^{3}$. Bien que cet effet doive être replacé dans son contexte, ce reportage joue un rôle non négligeable dans le processus qui fait accéder la question des conditions de travail dans les entreprises utilisatrices d'amiante au statut de problème nécessitant une intervention politique et administrative.

« Même dans les entreprises qui étaient censées, disons comme E, être les plus à même de prendre les dispositions les plus adaptées pour limiter le risque, la situation n'était pas fameuse en termes de prévention des risques professionnels, hein, certains montages... Certains films tournés sur place, qui montraient les condi-

1. Après l'interview d'un ouvrier affirmant que les chargements d'amiante se font manuellement avec ouverture de sac au couteau, donc en contact direct avec la personne, le commentaire du journaliste dénonce: «Des pratiques inacceptables dénoncées par tous les spécialistes à qui nous avons montré ces images. Les patrons de l'usine nous ont promis que des travaux allaient être entrepris prochainement. Une promesse faite régulièrement depuis vingt ans » («Envoyé spécial », France 2, 28 septembre 1995).

2. « Envoyé spécial », France 2, 28 septembre 1995.

3. Ces interventions de contrôle ont lieu très peu de temps après la diffusion du reportage, entre le 17 octobre et le 14 novembre 1995. La liste des infractions à la législation de 1977 relevées lors de ces visites montre l'inapplication effective de cette réglementation. La principale nouveauté de cette intervention de l'inspection du travail ne consiste toutefois pas dans le constat de ces infractions, qui sont courantes dans les domaines de l'hygiène et de la sécurité et relativement tolérées par les organes de contrôle (Emmanuel Henry, «Intéresser les tribunaux à sa cause. Contournement de la difficile judiciarisation du problème de l'amiante », Sociétés contemporaines, 52, 2003 p. 39-59). Elle tient plutôt à la nouvelle attitude de l'administration de contrôle vis-à-vis de ces infractions. Celles-ci deviennent inacceptables et font l'objet, pour certaines d'entre elles, d'une mise en demeure alors qu'en temps normal, elles font plutôt l'objet de négociations ou d'accommodations, la sanction n'intervenant qu'exceptionnellement, cf. Nicolas Dodier, «Les actes de l'inspection du travail en matière de sécurité : la place du droit dans la justification des relevés d'infraction», Sciences sociales et santé, 6 (1), février 1988, p. 8-28 et «Le travail d'accommodation des inspecteurs du travail en matière de sécurité », dans Luc Boltanski, Laurent Thévenot (dir.), Justesse et justice dans le travail, Paris, PUF, 1989 (Cahiers du centre d'études de l'emploi. 33), p. 281-306. 


\section{Emmanuel Henry}

tions dans lesquelles les gens ouvraient les sacs d'amiante et les vidaient dans des broyeurs ou dans des malaxeurs, nous laissaient quand même perplexes sur les conditions d'utilisation et les mesures qui pouvaient être faites de l'empoussièrement. » 1

L'importance acquise par ce reportage ne doit pas être analysée simplement comme la révélation d'une situation problématique aux acteurs directement chargés de sa gestion. Elle est plutôt symptomatique de la méconnaissance des questions de santé au travail de la part d'une majorité d'acteurs politiques - et de journalistes. Cette méconnaissance les conduit à aborder ces problèmes sur le même mode que les questions de santé publique à destination de la population générale, qu'ils maitrisent mieux. Pourtant, la gestion du risque professionnel encadre l'exposition d'une population ciblée à un risque connu, alors que le traitement des questions de santé publique touchant la population générale s'effectue plutôt en termes d'éradication de tout risque. Appliquer les cadres d'analyse propres aux questions de santé publique à destination de la population générale sur des questions de santé au travail aboutit à une situation où l'on met en évidence un risque dans une approche où, justement, tout risque doit disparaître. Les épisodes de forte publicisation des choix en matière de risque professionnel fragilisent donc leur acceptation sociale, qui repose précisément sur leur méconnaissance. Par l'intermédiaire de certains produits ou procédés utilisés à la fois professionnellement et dans la vie courante, comme l'amiante, certaines dimensions de ces arbitrages se trouvent de fait abordées dans le débat public, alors que les acteurs, qui encadrent ce débat ou qui doivent y répondre, méconnaissent les enjeux qui structurent ces problèmes. La discussion s'en trouve tronquée puisque cette contradiction des cadres d'approche rend immédiatement inacceptables les conséquences de ces choix. Celles-ci ne peuvent ainsi être abordées que sur le mode de la dénonciation, par certains journalistes véritablement surpris et logiquement indignés de ce qu'ils découvrent, ou par les acteurs associatifs et syndicaux qui cherchent ainsi à modifier en leur faveur un rapport de force ${ }^{2}$. Symétriquement, ils obtiennent une réponse de la part du personnel politique, en termes d'interdiction ou de traitement d'un scandale, donnant ainsi un statut d'exception à l'amiante, aux éthers de glycol et aux quelques toxiques professionnels ayant eu accès à l'espace public, alors que les types d'arbitrages desquels leur usage professionnel est issu n'ont rien d'exceptionnel et constituent plutôt une norme dans les secteurs sociaux qui traitent quotidiennement de ces questions. Pour le dire autrement, si l'amiante constitue un scandale de santé publique, alors c'est l'ensemble des toxiques professionnels qui pourrait être redéfini de cette manière. La nouvelle attention du personnel politique tranche pourtant radicalement avec le désintérêt antérieur porté à ces problèmes assez techniques et peu susceptibles de recevoir de forts échos publics. Elle est un facteur important de la priorité donnée à ce problème au sein de l'administration et de la vitesse avec laquelle il est traité.

«C'est vrai qu' on a toujours culturellement admis que le risque était supérieur en milieu de travail, c'est-à-dire que quand les gens descendaient à la mine, on n'a jamais dit il faut pas descendre à la mine parce qu'il y a des coups de grisou, on

1. Entretien avec un membre du cabinet de Jacques Barrot au ministère du Travail et des Affaires sociales, inspecteur général des affaires sociales, 8 février 1999.

2. C'est aussi ce que constate Denis Duclos : «En cette matière (peut-être plus que pour d'autres objets de revendication plus classiques du combat syndical), les "grands progrès" semblent liés à la capacité du mouvement social dans son ensemble à créer des "effets de scandale", auxquels concourent des combinaisons d'acteurs les plus divers » (Denis Duclos, La santé et le travail, Paris, La Découverte, 1984 (Repères), p. 86). 
a toujours accepté un risque en milieu de travail. Il y a un risque bien évidemment supérieur en milieu de travail qu'en milieu de populations. Et, si vous voulez, ce qui pèse sur nous, en ce moment, c'est que de façon non dite, on remet en cause cette pratique. [...] Et l'affaire du sang contaminé pèse tellement fort que y compris les politiques ne voient pas ce problème, c'est-à-dire qu'ils voient, ils nous disent, mais attention, les éthers de glycol... bon, le plomb, toxique pour la reproduction, on n'a pas totalement interdit le plomb. ${ }^{1}$

Sur un plan interne à l'administration, l'apparition de l'amiante dans les discours médiatiques a surtout des effets indirects. Elle offre une fenêtre d'opportunité tant pour les acteurs associatifs et syndicaux que pour les fonctionnaires du ministère ${ }^{2}$. La possibilité de s'appuyer sur la problématisation en termes de scandale promue par les discours médiatiques permet une modification du rapport de force entre les différents acteurs participant aux négociations, en donnant un poids supplémentaire aux acteurs associatifs et syndicaux. Toutefois, si l'intérêt des acteurs directement mobilisés est assez évident, il faut souligner que l'existence de cette fenêtre est aussi importante pour les acteurs de l'administration. Elle leur permet de faire avancer le dossier de façon plus rapide et prioritaire qu'ils n'auraient pu le faire autrement ${ }^{3}$. En modifiant l'équilibre des rapports entre acteurs, elle rend plus difficile une levée d'opposition franche aux décisions en préparation.

«On n'était pas absolument mécontents de voir que cette pression médiatique pouvait, à titre assez accessoire, avoir quand même un petit rôle pour aider à accélérer éventuellement un peu les choses. Elle a aussi modifié la perception des acteurs qui participent à ce processus. ${ }^{4}$

La publicité donnée au problème modifie aussi considérablement l'équilibre des rapports de force en faisant directement peser une menace sur les acteurs nettement mis en cause dans la définition publique du problème. Si, dans le cadre de négociations relativement confidentielles entre patronat et syndicats, tenir une position ferme d'opposition à toute avancée de la réglementation en matière de pathologie professionnelle peut avoir une certaine légitimité sociale (elle fait partie des règles du jeu entre partenaires sociaux), il n'en va pas du tout de même lorsque ce type de prise de position peut acquérir une dimension publique et, a fortiori, dans un contexte où l'usage de l'amiante est publiquement défini comme un scandale de santé publique. La publicité ou le risque qu'une publicité soit donnée aux débats et aux négociations contribue à invalider certaines prises de position, en particulier pour des scientifiques qui veulent garder une certaine crédibilité vis-à-vis de leurs pairs ${ }^{5}$.

1. Entretien avec le chef du Bureau hygiène en milieu de travail, Direction des relations du travail, 13 janvier 1999.

2. «The policy window is an opportunity for advocates of proposals to push their pet solutions, or to push attention to their special problems [la fenêtre d'opportunité est une occasion, pour les avocats d'une cause, de mettre en avant leurs solutions de prédilection ou d'attirer l'attention sur leurs problèmes particuliers] » (John W. Kingdon, op. cit., p. 173).

3. On retrouve ici une certaine similitude avec les analyses de Calliope Spanou dans les domaines de l'écologie, du féminisme et de la consommation, lorsqu'elle décrit les relations entre l'administration et les mouvements, mêlées de méfiance et de concurrence. Cf. Calliope Spanou, Fonctionnaires et militants. L'administration et les nouveaux mouvements sociaux, Paris, L'Harmattan, 1991 (Logiques politiques), p. 187 et suiv.

4. Entretien avec le sous-directeur chargé des conditions de travail, Direction des relations du travail, 24 mars 1999.

5. Cf., dans le même sens, sur la question des lombalgies professionnelles, Marc-Olivier Déplaude, art. cité. 


\section{Emmanuel Henry}

Le processus de publicisation affecte donc différemment la DRT et la DGS dans l'élaboration de la réglementation concernant l'amiante. Si, à la DGS, c'est l'anticipation d'un risque de crise publique qui pousse à chercher des solutions au problème, dans le cas de la DRT, la publicité agit d'une triple manière. Elle invalide tout d'abord certaines prises de position, en particulier celles de certains experts qui ne peuvent plus légitimement siéger aux côtés des représentants patronaux, dans un contexte où existe un risque que leur prise de position soit rendue publique et où le problème est défini comme un «scandale de santé publique ». Ensuite, la publicité donnée aux connaissances scientifiques à partir desquelles les décisions sont prises modifie considérablement l'état des rapports de forces entre les différents intervenants. Les acteurs associatifs et syndicaux peuvent dorénavant appuyer leurs demandes sur une expertise qui fait l'objet d'un consensus et ne risque donc plus d'être invalidée par une rafale de contre-études toujours mobilisables sur une question controversée ; les représentants patronaux ont perdu la capacité de réfuter certaines données, une fois celles-ci publiquement admises, énoncées et diffusées. Enfin, les acteurs administratifs, soutenus par une volonté politique affichée, ont la capacité de jouer un rôle plus actif et plus clair vis-à-vis des représentants patronaux, dans les arbitrages effectués.

\section{L'INTERVENTION POLITIQUE : UNE RÉPONSE AU PROBLÈME PUBLIC}

Parallèlement au travail administratif précédemment analysé, la crise de l'amiante se développe dans les médias d'information comme un scandale de santé publique et contraint le personnel politique à prendre position et à annoncer un certain nombre de décisions. Si l'on suit le développement public du problème, on remarque que deux décisions politiques sont unanimement présentées comme les solutions au problème. Elles interviennent, toutes les deux, en juillet 1996, à un moment où l'amiante apparaît comme une affaire mettant particulièrement en cause l'État dans son rôle de protection des populations. Elles suivent de près le dépôt d'une plainte par l'Association nationale de défense des victimes de l'amiante (ANDEVA), le 25 juin 1996, et l'annonce des premiers résultats de l'expertise collective menée par l'INSERM, le 3 juillet 1996. Ainsi, ce même jour, Jacques Barrot, ministre du Travail et des Affaires sociales, annonce que « la fabrication, l'importation et la mise en vente de produits contenant de l'amiante [...] sont interdits ». Quelques jours plus tard, au cours de la « Garden party » du 14 juillet, Jacques Chirac, président de la République, déclare en direct sur TF1 et France 2 : «Avant la fin de l'année, il n'y aura plus d'étudiants à Jussieu » ${ }^{1}$. Ces deux déclarations sont les réponses politiques les plus fortes apportées au problème posé par l'amiante, tel qu'il est publiquement formulé depuis 1995 ; elles apparaissent, au personnel politique qui y est confronté, comme les solutions les plus adaptées au problème qu'il rencontre. Ce sont aussi les décisions politiques qui ont le plus d'impact public et médiatique et contribuent fortement à la résolution de la situation publique de crise.

1. La phrase complète prononcée par Jacques Chirac lors de cette conférence de presse est : « Naturellement, on ne va pas continuer à enseigner à Jussieu, il va y avoir le temps du déménagement, mais avant la fin de l'année, il n'y aura plus d'étudiants à Jussieu, parce qu'il y a un risque. Cela implique un coût, mais ce sont des coûts qu'il est nécessaire d'assumer », $L e$ Monde, 16 juillet 1996. 
L'impact public et médiatique d'une décision politique, s'il n'est pas totalement prévisible, est tout de même l'objet d'anticipations de la part du personnel politique et la facilité avec laquelle celui-ci se laisse imposer une définition du problème en termes environnementaux conduit à supposer que cette problématisation n'est pas totalement antinomique avec les intérêts qu'il cherche à privilégier. En laissant l'attention du public se focaliser sur la dimension la plus périphérique du risque, à laquelle il est relativement aisé de trouver une solution satisfaisante, les acteurs politiques rendent possible une résolution de la crise autour de quelques décisions spectaculaires pouvant être facilement reconnues et comprises. À l'inverse, si les questions liées au risque professionnel étaient apparues comme le cœur du problème, il aurait sans doute été beaucoup plus complexe d'aboutir au même effet d'affichage, étant donné la technicité des réponses apportées dans ce domaine.

\section{LA PRÉSENCE DU POLITIQUE DÈS LE DÉBUT DE LA « CRISE »}

Le premier écueil à l'analyse des situations critiques serait de placer le personnel politique dans la situation de devoir répondre à une crise qui se constituerait en dehors de son action ${ }^{1}$. Or, on constate justement l'inverse puisque, dès les premières redéfinitions publiques de la question de l'amiante, fin mai 1995, Élisabeth Hubert, ministre de la Santé récemment nommée, intervient en première ligne. Elle annonce, dans une interview au journal de 20 heures de TF1 du 31 mai, relayée le lendemain dans Le Monde, la parution prochaine des décrets qui rendront obligatoire la recherche d'amiante dans les bâtiments, dont nous avons vu précédemment les logiques administratives d'élaboration. Le fait que la ministre intervienne et ne «mâche pas ses mots $»^{2}$ est un des éléments qui contribuent à imposer l'amiante comme un problème de premier plan.

Contrairement aux lectures journalistiques qui en sont faites, cette prise de position ne peut pas être analysée comme la réponse donnée de l'extérieur à un problème public en pleine émergence. Tout d'abord, à l'inverse, cette forte intervention politique est un des facteurs qui contribuent à situer ce problème à la une des médias d'information et, ce faisant, à le constituer en crise. Ensuite, les décrets annoncés sont un bon exemple d'une solution administrative en cours d'élaboration qui trouve un problème public à sa mesure pour permettre l'affichage d'une volonté politique forte ${ }^{3}:$ ils ont fait, comme on l'a vu, l'objet d'un lent processus administratif d'élaboration et sont

1. Comme l'indique Claude Gilbert : «Un premier biais consiste à situer les "politiques" a priori en position d'extériorité. [...] En fait, les "politiques" ne sont pas "face à la crise", mais dans la crise, au même titre que d'autres acteurs, et il n'est pas rare qu'ils soient eux-mêmes des activateurs de ces crises» (Claude Gilbert, "Risques sanitaires et sciences humaines et sociales : quelques pistes de recherche », Revue française des affaires sociales, 1, janviermars 1999, p. 17).

2. «Élisabeth Hubert, le ministre de la Santé publique, n'a en tout cas pas mâché ses mots, mercredi 31 mai après le conseil des ministres : "Cela représente des crédits à dégager, mais avec la sécurité des populations, on ne doit pas badiner de quelque façon que ce soit" ", Le Monde, 2 juin 1995 [italique de citation des auteurs].

3. Ces solutions en quête de problème sont assez proches des « solutions en quête de questions auxquelles elles pourraient répondre » décrites dans le cas des organisations par Johan P. Olsen, James G. March, cité, p. 165. 
en voie de finalisation lorsque la ministre prend la parole sur le dossier. Un autre regard, interne à l'administration, montre bien cet aspect du processus.

«Quand Mme Veil et M. Douste-Blazy s'en vont, c'est à l'occasion de l'élection présidentielle, juin 95, le décret est pratiquement fini. Bon, Mme Hubert arrive, je le lui vends, je me rappelle très bien, elle annonce avec puissance qu'elle a demandé un décret, qui est pratiquement fait, bon et puis après, il entre en application en 1996. ${ }^{1}$

Même si les deux séries d'événements - élaboration du décret sur le recensement des bâtiments contenant de l'amiante et montée en puissance du problème public sont indépendantes l'une de l'autre, leur convergence est immédiate. Les journalistes, confrontés à ce qu'ils définissent comme un problème relevant du ministère de la Santé, s'adressent logiquement à Élisabeth Hubert pour avoir des réponses aux questions qu'ils se posent, liées au risque concernant l'ensemble de la population. Ils sont ainsi consacrés et renforcés dans leur rôle social puisque leur dénonciation du problème est couronnée de succès. Dans le même temps, la ministre de la Santé, en présentant sa solution qui cherchait justement le problème public auquel s'appliquer, trouve dans la définition médiatique un moyen d'affirmer une capacité à agir qui rejaillit sur l'ensemble du gouvernement. L'adéquation entre l'interrogation des journalistes et la solution existant au ministère de la Santé présente, en outre, l'avantage de ne pas immédiatement aborder l'essentiel des problèmes posés par l'amiante, liés à son utilisation professionnelle, vis-à-vis desquels le ministère du Travail se trouve dans une position moins facile, le processus d'élaboration des décrets relevant de sa compétence n'étant pas encore enclenché. En acceptant de se laisser imposer une définition en termes de scandale de santé publique, les acteurs politiques se mettent évidemment en situation de risque, mais ils se placent aussi face à un problème auquel ils peuvent immédiatement répondre. Ils montrent qu'ils savent prendre leurs « responsabilités » à un moment où c'est justement l'absence de décision qui leur est directement reprochée.

L'annonce de l'interdiction de l'amiante se situe dans la même logique. Elle est « faite sur mesure » par le personnel politique pour répondre aux principales préoccupations relayées par les médias d'information ; elle apparaît d'emblée à l'ensemble des journalistes comme la mesure indispensable, voire suffisante, pour résoudre le problème : ils la relaient alors amplement.

\section{L'INTERDICTION DE L'AMIANTE : UNE DÉCISION DÉFINITIVE ?}

Dire de l'interdiction de la fabrication, de l'importation et de la mise en vente des produits contenant de l'amiante, annoncée le 3 juillet 1996, qu'elle constitue une réponse particulièrement en phase avec la problématisation publique ne signifie pas pour autant que cette décision est purement de l'ordre du symbolique ${ }^{2}$. Elle entre pleinement dans la stratégie de résolution des problèmes posés par l'amiante, mais elle n'est qu'une condition de son règlement à long terme : elle ne modifie en rien la situation actuelle.

1. Entretien avec le directeur général de la santé de 1986 à 1997, 24 novembre 1998.

2. Cf. Murray Edelman, Pièces et règles du jeu politique, Paris, Le Seuil, 1991 (La couleur des idées) (1 $1^{\text {re }}$ éd. américaine : 1988), qui tend à promouvoir ce type d'analyses. 
L'interdiction de l'amiante se ramène en pratique essentiellement à l'interdiction de l'amiante-ciment, qui représente en 1996 le débouché de $95 \%$ des 35000 tonnes d'amiante importées en France; les autres utilisations de l'amiante ont quasiment toutes été abandonnées avec les restrictions de mise sur le marché successives imposées par la réglementation européenne. L'interdiction a pour principal effet de faire passer d'une situation de mise sur le marché extrêmement réglementée à celle d'une interdiction avec des exceptions et ne constitue donc pas un changement majeur sur le plan des politiques publiques en vigueur. Seules les modalités de mise en œuvre sont modifiées : le choix de l'interdiction avec exceptions fait passer d'une situation de contrôle a posteriori des matériaux produits à un dispositif de vérification préalable des dérogations par l'administration.

Ainsi, ce qui est présenté par les discours d'information médiatiques comme la décision politique majeure vis-à-vis de l'amiante, parce qu'elle répond exactement à une exigence explicitement exprimée à plusieurs reprises par les journalistes, peut être analysé au sein de l'administration comme une simple volonté d'affichage de la part du personnel politique.

«Pour moi, clairement, l'amiante-ciment devait être éliminé. Le cabinet est allé beaucoup plus loin et a pris une mesure d'interdiction totale, qui revient au même puisqu'elle est basée sur le principe : s'il existe une substitution, on n'a pas le droit d'utiliser l'amiante, mais qui nous a obligés à mettre en place un système assez complexe, qu'on continue à gérer, de gestion des exceptions à l'interdiction. » ${ }^{1}$

L'interdiction est immédiatement présentée comme une solution définitive par l'ensemble des médias. Le Monde titre le 4 juillet 1996 : «La France sera le huitième pays européen à interdire l'amiante »; l'article débute ainsi : « Le ministre du Travail et des Affaires sociales, Jacques Barrot, a annoncé, mercredi 3 juillet, l'interdiction définitive de l'amiante sur le territoire français. La France devient ainsi le huitième pays d'Europe, avec l'Allemagne, l'Italie, le Danemark, la Suède, les Pays-Bas, la Norvège et la Suisse, à bannir cette fibre minérale dont le caractère cancérogène a été mis en évidence depuis au moins trente ans. » Si le retard de la France est nettement critiqué, la décision prise est analysée comme un élément essentiel du règlement du problème. Face à ce qui apparaît dans les discours d'information comme un risque immédiat, accrédité par les témoignages douloureux de victimes, l'interdiction est la seule solution capable de rendre supportable l'exhibition de leur souffrance, puisque censée pouvoir y mettre fin. C'est essentiellement autour de l'annonce de cette décision que peut se construire une normalisation du problème public. Le 3 juillet 1996, le premier titre du journal de 20 heures de France 2 reprend cette information

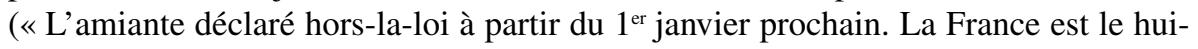
tième pays à prendre cette décision ${ }^{2}$ ), alors que le présentateur de celui de TF1 indique : «Jacques Barrot a décidé d'interdire la production et la mise en vente de

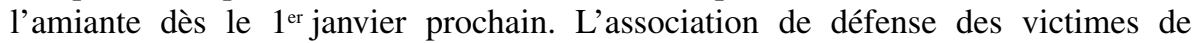
l'amiante se félicite de cette mesure. »

À propos de la comparaison européenne qui, dans les discours médiatiques, désigne le retard de la France comme particulièrement marqué, il faut souligner que la

1. Entretien avec le chef du bureau hygiène en milieu de travail, Direction des relations du travail, 13 janvier 1999.

2. La première phrase d'ouverture du journal après les titres est la suivante : « Madame, Monsieur, bonsoir. La fabrication, l'importation et la mise en vente de produits contenant de l'amiante seront désormais interdites. La mesure prendra effet au $1^{\text {er }}$ janvier prochain ». 


\section{Emmanuel Henry}

plupart des décisions d'interdiction totale avec dérogations dataient du courant des années 1990 et que la distance peut être très mince entre utilisation réglementée et interdiction avec dérogations. En situant l'analyse à un niveau plus fin que l'alternative interdiction/autorisation, on se trouve face à une situation beaucoup plus contrastée. Pour l'amiante-ciment, principal mode d'utilisation de l'amiante, si les pays scandinaves l'ont interdit dès les premières années de la décennie 1990, voire quelques années avant ${ }^{1}$, les dérogations qui le concernaient sont arrivées à échéance en 1994 et 1995 pour l'Allemagne, l'Italie et l'Autriche ${ }^{2}$. En interdisant l'amiante au premier janvier 1997, la France quitte bien sa logique antérieure de défense de l'utilisation de l'amiante, mais le contraste avec les autres pays européens est moins net que ce que laissent penser les discours médiatiques d'information. Ces discours, qui opposent en Europe les pays «abolitionnistes » et les pays «permissifs », insistent donc sur le caractère symbolique ou emblématique ${ }^{3}$ d'une politique publique, en méconnaissant largement son application effective.

Pour comprendre comment l'interdiction s'impose comme une solution possible auprès du personnel politique, il faut souligner que le poids économique de la filière industrielle amiante a considérablement diminué depuis vingt ans. Les deux principaux groupes industriels produisant des matériaux à base d'amiante, Saint-Gobain (au sein de ses filiales Everite et Pont-à-Mousson) et Eternit n'emploient plus que deux mille salariés en 1995, contre quinze mille dans les années 1970. L'ensemble du secteur industriel des produits contenant de l'amiante ne représente que quatorze entreprises et emploie trois mille cinq cents personnes ${ }^{4}$. Comme le constatent les auteurs du rapport parlementaire sur l'amiante : «Il saute aux yeux que, d'un point de vue macro-économique, l'effet de la décision d'interdiction est des plus limité » ${ }^{5}$. L'interdiction est donc une décision que le pouvoir politique peut prendre relativement facilement. Elle entre pleinement dans ses attributions et ne constitue pas véritablement un enjeu puisque aucune opposition forte ou structurée ne s'est vraiment manifestée, les industriels se situant dans une optique de reconversion à long terme, déjà effectuée dans les pays aux réglementations plus strictes. L'analyse de la décision d'interdiction proposée par les parlementaires est assez lucide sur ses impacts réels. Elle en souligne néanmoins l'enjeu principal : envoyer un signal fort à l'opinion publique. La nécessité de décider politiquement de l'interdiction de l'amiante leur apparaît explicitement comme une réponse au problème public, «une décision qui concerne l'opinion publique et qui a pour effet de la rassurer » :

«C'est pour avoir tardé à apporter une réponse définitive que le dossier de l'amiante s'inscrit aujourd'hui dans un tel contexte médiatique et que l'opinion publique a pu en être fortement déroutée. [...] La décision politique d'interdiction consiste globalement à mettre en place toutes les mesures nécessaires afin de

1. Les dernières dérogations pour la production d'amiante-ciment arrivaient à échéance en 1988 pour le Danemark, 1992 pour la Suède et 1993 pour la Finlande et les Pays-Bas.

2. Cf. sur ce point, OPECST, «L'amiante dans l'environnement de l'homme... », rapport cité, p. 52-55, et Comité économique et social de l'Union européenne, «Avis du Comité économique et social sur l'amiante », Journal Officiel des Communautés européennes, C138, p. 26.

3. Pour reprendre le terme employé par Alexandre Siné au sujet de certains aspects des politiques budgétaires (Alexandre Siné, "L'ordre budgétaire. L'économie politique des dépenses de l'Etat », thèse de doctorat, Cachan, École normale supérieure de Cachan, 2002).

4. Cf. OPECST, «L'amiante dans l'environnement de l'homme... », rapport cité, p. 5657.

5. Ibid., p. 57. 


\section{Résoudre la crise de l'amiante?}

garantir, de protéger la santé et le bien-être des travailleurs exposés à l'amiante et de la population au sens large, et de réduire ainsi le risque à un niveau acceptable. ${ }^{1}$

L'interdiction de l'amiante, malgré son impact immédiat limité, est ainsi parée de tous les attributs d'une décision « définitive ». Du point de vue des acteurs politiques, le choix de privilégier l'interdiction constitue pourtant principalement un choix par défaut : elle est la seule réponse disponible à la seule partie du problème sur laquelle il est possible d'agir de façon spectaculaire. Elle constitue tout d'abord une réponse facile à mettre en œuvre dans le cadre de routines administratives éprouvées : décréter l'interdiction, définir les exceptions restent un travail d'élaboration de textes administratifs relativement classique. Le contrôle de sa mise en œuvre relève ensuite de la Direction générale de la concurrence et de la répression des fraudes, donc du ministère de l'Économie et des Finances, mieux doté que le ministère du Travail. Cette décision peut enfin s'expliquer simplement : il est beaucoup plus efficace de communiquer sur l'interdiction que sur les différentes modalités de protection des personnels exposés professionnellement aux poussières d'amiante.

La disproportion entre l'impact médiatique de cette décision et son impact réel est frappante. Avant et après cette annonce, les problèmes posés par l'amiante restent sensiblement les mêmes puisque les expositions des professionnels du bâtiment perdureront tant que ce matériau sera présent, soit pendant encore plusieurs décennies. Or, ces dimensions du problème, méconnues de nombreux acteurs sociaux, sont restées largement hors champ de sa définition médiatique et apparaissent donc avec moins d'acuité. Elles peuvent faire l'objet d'un traitement à plus long terme puisque leur moindre visibilité leur assure une plus grande acceptation sociale.

Le problème plus circonscrit du site de Jussieu se présente différemment, car il est l'objet d'une attention prioritaire de la part des médias d'information. Dans un contexte de succession d'affaires de santé publique, la dénonciation d'un risque à Jussieu exerce une pression sur les acteurs politiques afin qu'ils interviennent et annoncent des décisions importantes. Ce n'est que dans cette perspective que l'intervention de Jacques Chirac annonçant la fermeture du campus dans l'année peut recevoir un début d'explication. Elle correspond à une modalité d'exercice du pouvoir qui pousse à l'extrême l'utilisation de l'efficace propre de la parole politique, presque indépendamment de la décision réelle annoncée, dans ce cas, totalement irréaliste ${ }^{2}$. Le fait que le premier personnage de l'État prenne position sur le problème et le consacre comme prioritaire apparaît plus fondamental que le contenu de ce qu'il annonce. Cette prise de parole est donc avant tout une réaffirmation de la capacité du politique à agir sur le cours des choses et à le modifier, qui le place en pleine conformité avec le rôle social qui lui est assigné ${ }^{3}$.

1. Ibid., p. 57-58 [souligné par moi].

2. Sur l'importance de la parole dans l'exercice du pouvoir, cf. Pierre Clastres, La société contre l'État, Paris, Minuit, 1974 (Critique). Cf. aussi Patrick Lehingue, « La parole présidentielle. Travail de codification et définition du poste », dans Bernard Lacroix, Jacques Lagroye (dir.), Le président de la République. Usages et genèses d'une institution, Paris, Presses de Sciences Po, 1992, p. 109-140.

3. C'est depuis l'anthropologie qu'est le mieux analysée cette dimension centrale du pouvoir politique, cf., par exemple, Georges Balandier, Le pouvoir sur scène, Paris, Balland, 1992 (Fondements). Cf. aussi Jacques Lagroye, «La légitimation », dans Madeleine Grawitz, Jean Leca (dir.), Traité de science politique. 1. La science politique, science sociale. L'ordre politique, Paris, PUF, 1985, p. 395-467. Sur la question des rôles politiques, cf. les différents articles réunis dans les numéros de Politix, « Le métier d'élu. Jeux de rôles », 28, 4e trim. 1994, et «L'institution des rôles politiques », 38, 2 trim. 1997. 


\section{Emmanuel Henry}

Alors que l'essentiel des problèmes posés par l'amiante a déjà fait l'objet d'un règlement politico-administratif avec les décrets de février 1996, la résolution de la crise publique s'effectue autour d'une autre temporalité et avec d'autres acteurs. La normalisation des discours publics sur l'amiante n'a lieu qu'en juillet 1996, autour de quelques décisions emblématiques prises par le personnel politique. Si ces décisions répondent plus directement au problème tel qu'il est formulé publiquement qu'aux difficultés réelles que pose l'utilisation massive de l'amiante depuis un demi-siècle, elles ne doivent pas amener simplement à conclure sur la forte interdépendance entre personnel politique et médiatisation du problème ou à souligner les conséquences de cette interdépendance en termes de spectacularisation de la politique. Cette forme de réponse politique aux problèmes critiques doit plutôt être analysée comme une contrainte à « endosser » le pouvoir, selon le terme employé par Claude Gilbert dans l'analyse des situations de crise ${ }^{1}$. On retrouve, en effet, dans la résolution de cette crise par le politique, le même type de mécanismes que ceux analysés par cet auteur dans le cas des crises post-accidentelles. L'intervention apparaît comme la restauration d'une image classique du pouvoir politique, comme celui qui peut agir sur une situation et résoudre les problèmes posés par elle. Face à une crise ayant pris une telle envergure, ce n'est que le représentant du pouvoir politique à son plus haut niveau qui doit intervenir et il ne peut le faire que par des décisions spectaculaires et présentées comme définitives ; l'interdiction de toutes les formes d'amiante sur le territoire et l'annonce de la fermeture du campus de Jussieu sont des réponses de cet ordre ${ }^{2}$.

L'analyse du traitement politico-administratif de la crise de l'amiante dans un contexte de forte publicité ne peut donc pas se ramener à une relation causale univoque entre des mobilisations et une médiatisation, d'une part, et, d'autre part, l'intervention d'instances politiques et administratives contraintes à prendre en charge le problème. La diversité et la complexité des processus en jeu amènent à restituer un tableau beaucoup plus nuancé. La résolution politico-administrative de la question de l'amiante doit plutôt être analysée comme un ensemble de zones d'ombre et de lumière qui peuvent être dégagées selon une double perspective. La première met en évidence un continuum entre des logiques de mise sur agenda politico-administratif relativement autonomes et les conséquences directes d'une forte mobilisation ou d'une intense médiatisation ${ }^{3}$. La décision de recensement des bâtiments contenant de l'amiante, annoncée le 31 mai 1995 par la ministre de la santé, se rapproche de la première extrémité puisque, si le problème prend une dimension publique importante avec cette

1. Claude Gilbert, Le pouvoir en situation extrême. Catastrophes et politique, Paris, L'Harmattan, 1992 (Logiques politiques). Cf. aussi Claude Gilbert (dir.), La catastrophe, l'élu et le préfet, Grenoble, Presses universitaires de Grenoble, 1990.

2. «Le traitement de la crise passe par la mobilisation d'un arsenal classique, voire archaïque, du pouvoir politique, qui finit toujours par endosser des décisions radicales restaurant une réalité décidable et largement pensée à travers le modèle traditionnel de la guerre, qui retrouve alors toute son efficacité : au phénomène des crises "sans ennemi", il est finalement répondu par un retour du politique dans une de ses modalités les plus anciennes » (Claude Gilbert, Le pouvoir en situation extrême..., op. cit., p. 211, italiques de l'auteur).

3. Sur ce point, cf. John W. Kingdon, op. cit. ; Philippe Garraud, «Politiques nationales : élaboration de l'agenda », op. cit., ou Pierre Favre, "L'émergence des problèmes dans le champ politique », dans Pierre Favre (dir.), Sida et politique. Les premiers affrontements (19811987), Paris, L'Harmattan, 1992 (Dossiers sciences humaines et sociales), p. 5-37. 
annonce, il était pris en charge dans le cadre de routines administratives depuis la fin des années 1980. Ainsi, les décrets de février 1996, qui officialisent cette décision, bien qu'ils fassent l'objet d'un fort affichage politique, sont avant tout le résultat d'une gestion routinière du problème de la part de l'administration de la santé. À l'inverse, la décision de l'interdiction de l'amiante peut être analysée comme un effet assez direct de la forte pression médiatique sur le personnel politique pour qu'il intervienne vigoureusement sur le dossier. Si la limite entre mise sur le marché réglementée et interdiction avec dérogation de l'amiante est ténue, l'annonce de la décision d'interdiction n'en apparaît pas moins comme la décision qu'il était nécessaire de prendre face à l'envergure publique prise par le problème. La répercussion médiatique de cette décision est d'ailleurs à la mesure de son adéquation à la formulation la plus publique de cette crise. Elle nous amène au deuxième critère de différenciation entre zones d'ombre et de lumière du processus de gestion politico-administrative. Au-delà de la différenciation entre les modalités de mise sur agenda, une deuxième distinction s'impose. Parmi les décisions prises sur la période, toutes n'ont pas la même éligibilité à la publicité. Ainsi, les deux décrets « santé » et « travail » qui paraissent le 7 février 1996 ne suscitent pas du tout le même suivi public. Le premier, attendu par les médias d'information, fait l'objet d'une couverture assez importante ${ }^{1}$ et apparait comme un élément important dans le règlement du problème. Le second, en revanche, reste publiquement marginalisé, alors que ses conséquences sont sans commune mesure du point de vue des effets sanitaires des expositions à l'amiante. Conformément à la problématisation centrée sur la dimension environnementale, c'est globalement la majeure partie de la réglementation de l'usage professionnel du matériau qui est ainsi occultée des discours publics et médiatiques. À l'inverse, toutes les décisions en adéquation avec la définition du risque amiante comme touchant toute la population sont largement couvertes, à l'instar de l'annonce de l'interdiction de l'amiante ou de celle du déménagement de Jussieu.

Emmanuel Henry est maître de conférences à l'Institut d'études politiques de Strasbourg et membre du Groupe de sociologie politique européenne (GSPE). Dans le prolongement de sa thèse de doctorat, "Un scandale improbable. Amiante : d'une maladie professionnelle à une "crise de santé publique" », il a notamment publié «Intéresser les tribunaux à sa cause. Contournement de la difficile judiciarisation du problème de l'amiante », Sociétés contemporaines, 52, 2003, p. 39-59 ; «Le droit comme vecteur de publicisation des problèmes sociaux. Effets publics du recours au droit dans le cas de l'amiante », dans CURAPP, Sur la portée sociale du droit. Usages et légitimité du registre juridique, Paris, PUF, à paraître en 2004. Ses recherches portent actuellement sur les liens entre processus de publicisation, construction des problèmes publics et formes de l'action publique, notamment à partir de questions de santé publique.

1. L'article le plus complet sur ces décrets paraît dans Libération, le 14 février 1996. Titré «Amiante, pas une fibre ne doit rester. Un décret oblige les propriétaires à décontaminer les bâtiments touchés ", il ne mentionne même pas l'existence du second décret. 


\section{Emmanuel Henry}

\section{RÉSUMÉ/ABSTRACT}

QUAND L'ACTION PUBLIQUE DEVIENT NÉCESSAIRE: QU'A SIGNIFIÉ «RÉSOUDRE» LA CRISE DE L'AMIANTE ?

Cet article analyse l'influence de la forte publicité faite à un problème sur les modalités de sa gestion politique et administrative à partir du cas de l'amiante lors de son épisode de forte publicisation, en 1994-1996. La dimension la plus visible de ce processus est la contrainte à devoir agir de façon spectaculaire, imposée au personnel politique. Pourtant, cet aspect du processus ne doit pas masquer les logiques propres à certains secteurs administratifs dans leur gestion plus autonome du problème. L'analyse de ces logiques révèle que, si la publicisation joue un rôle, c'est à travers des prismes différents selon les secteurs administratifs concernés, tous n'étant pas sensibles de la même manière à la publicité.

\section{WHEN PUBLIC ACTION BECOMES NECESSARY : « RESOLVING » THE ASBESTOS CRISIS}

The article focuses on the asbestos crisis (between 1994 and 1996) with a view to analyzing the consequences of the strong publicity associated with this issue's political and management process. Most obvious in such a process was the necessity for political actors to act in a spectacular way. However this cannot cover the specific logic of certain administrative sectors in the management of the problem. The analysis of such logic demonstrates that in spite of publicity's influence each administrative sector concerned responded differently due to their own specificity and predisposition to publicity. 